\title{
A meso-mechanical model for concrete under dynamic tensile and compressive loading
}

\author{
L. Snozzi · F. Gatuingt · J.F. Molinari
}

Received: date / Accepted: date

\begin{abstract}
We present a computational model, which combines interface debonding and frictional contact, in order to investigate the response of concrete specimens subjected to dynamic tensile and compressive loading. Concrete is modeled using a meso-mechanical approach in which aggregates and mortar are represented explicitly, thus allowing all material parameters to be physically identified. The material phases are considered to behave elastically up to failure and the initiation, coalescence and propagation of cracks are modeled by dynamically inserted cohesive elements. The impenetrability condition is enforced by a contact algorithm that resorts to the classical law of Coulomb friction.

We show that the proposed model is able to capture the general increase in strength with increasing rate of loading and the tension/compression asymmetry. Moreover, we simulate compression with lateral confinement showing that the model reproduces the increase in peak strength with increasing confinement level. We also quantify the increase in the ratio between dissipated frictional energy and dissipated fracture energy as the confining pressure is augmented. Our results demonstrate the fundamental importance of capturing frictional mechanisms, which appear to dissipate substantially more energy than cracking under compressive loading.
\end{abstract}

Keywords Cohesive Zone Model · Dynamic Fracture · Concrete · Frictional Contact $\cdot$ Meso-scale $\cdot$ Numerical Methods

L. Snozzi · J.F. Molinari

ÉCole Polytechnique Fédérale de Lausanne (EPFL), School of Architecture, Civil and Environmental Engineering (ENAC), Computational Solid Mechanics Laboratory (LSMS), Bâtiment GC - A2, Station 18, CH 1015-Lausanne

Tel.: (+41) 216932411

Fax: (+41) 216936340

F. Gatuingt (corresponding author)

L.M.T. Cachan, 61 Avenue du Prsident Wilson, FR 94235-CACHAN Cedex

Tel: $(+33) 147407460$

Fax: (+33) 147402240

E-mail: gatuingt@1mt.ens-cachan.fr 


\section{Introduction}

Failure of heterogeneous (quasi) brittle materials such as concrete, is a complex and nonlinear phenomenon which dissipates energy according to its (meso-structural) composition, geometry and loading conditions. Fracture of these materials involves the opening of local micro-cracks, which may propagate, coalesce and subsequently enter into contact influencing the nonlinear failure process. Therefore, a careful treatment of both fracture and frictional mechanisms is needed in order to correctly reproduce the material's behavior.

The concrete constitutive behavior can be formulated either at the macro-scale or at the meso-scale. At the macro scale, the ingredients that characterize concrete's heterogeneity are not represented and one considers it as a homogeneous material. Therefore, in this case, the constitutive models need to have recourse to (visco)-

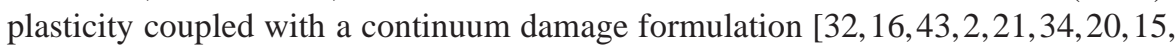
36 14]. This leads to models with a relatively high number of parameters, which are difficult to relate to physical mechanisms that occur during failure. On the contrary, a meso-scale level of observation (as proposed in [50]) allows an explicit representation of some concrete constituents, which enables reducing the number of model parameters and to describe the interactions between matrix and inclusions. In the literature one can find several meso-scale models for concrete. They can be divided in two main computational classes. A first family is represented by lattice models (for instance [42 4]), where the continuum is replaced by a system of discrete particles and the mechanical properties of the lattice beams aim to represent the concrete mesostructure [ [ $\left.\begin{array}{lllll}26 & 24 & 23 & 13\end{array}\right]$. The second class resorts to the finite-element approach, in which concrete is usually represented as a biphasic material, made of a mixture of aggregates embedded in a matrix phase with an interfacial transition zone (ITZ) between them [38 471029,964422$].$

The aim of this study is to develop a 2D meso-mechanical finite-element model, which couples together cohesive zone modeling for crack propagation with a contact algorithm to enforce the impenetrability condition in explicit dynamics. For this purpose, we extend the mesoscopic approach that we had already adopted in 44 . 22], in order to include compressive cracking capability (with the same formulation proposed in [45]). The main characteristics of this model are a continuous transition from decohesion to pure frictional sliding (note that the onset of friction starts in conjunction with the onset of cracking). The debonding is controlled by a novel initially-rigid traction separation law, which enables us to define two separate values for the dissipated fracture energy in mode I and II. In this paper we will verify if the approach is able to reproduce the dissymmetric tensile/compressive behavior, strain rate strengthening and confinement effects.

The paper is composed as follows. Section 2 describes the chosen finite element framework for representing compressive cracking at the interfaces. In Section 3 the meso-scale approach with its material parameters is presented. Results are reported in Section 4 for uniaxial tension and compression. While results on biaxial compressive loading are listed in Section 5 Finally, concluding remarks are stated in Section 6 


\section{Numerical Approach}

The following section summarizes the formulation of the adopted frictional/cohesive capability. A complete formulation and validation of the method can be found in [45]. The approach has been conceived for simulations in explicit dynamics (second order explicit version of the popular Newmark $\beta$-method [31]). This scheme is applied to the discretized equation of dynamic equilibrium:

$$
\mathbf{M} \ddot{\mathbf{x}}+\mathbf{R}^{\text {int }}=\mathbf{R}^{\mathbf{e x t}}
$$

where $\mathbf{M}$ represents the mass matrix, $\ddot{\mathbf{x}}$ the nodal acceleration vector and $\mathbf{R}^{\mathbf{e x t}}$ and $\mathbf{R}^{\text {int }}$ are the external and internal force vector respectively. The combination of a lumped mass matrix with the explicit scheme allows to trivially invert the mass matrix solving explicitly the scheme. Stability is achieved under the condition that the time step is below a critical value, which is

$$
\Delta t_{c r i t}=\alpha \min _{1 \leq e \leq N_{e}}\left(\frac{l_{e}}{c}\right)
$$

where $\mathrm{c}$ represents the plain strain compression stress wave speed and $l_{e}$ is the size of the element. The stable time step has to be chosen equal to the smallest value over all elements $\left(N_{e}\right)$ multiplied by a security coefficient $\alpha$ (typically around 0.1 ). All the simulations presented thereafter have been conducted in a $2 \mathrm{D}$ plane strain configuration using mesh composed of 6-noded quadratic triangles.

\subsection{Cohesive approach}

A well-known method to model the onset of fracture is to have recourse to cohesive zone modeling, which has been introduced by Dugdale [17] and Barenblatt [1] in the 1960s. This method describes fracture as a separation process by relating the displacement jump, which occurs at the crack tip, with tractions. The debonding is assumed to be confined in a small region of material called the cohesive zone, where atomistic separation occurs. Within the computational framework this region (also called fracture process zone) is represented by interface elements with null thickness. While damage is concentrated in these elements, we will assume that the surrounding bulk material behaves linear elastically. The decohesion process is controlled by a constitutive relationship called traction separation law (TSL), which is usually related to a potential. Depending on the response of the cohesive surface prior softening behavior it is possible to distinguish between two main classes of TSL. The interface can exhibit an initial elastic behavior, intrinsic approach (e.g. [51]), or is assumed to be initially rigid, extrinsic approach (e.g. [7]). This second method implies that cohesive elements have to be inserted dynamically, avoiding the artificial compliance of the uncracked body generated by having recourse to the intrinsic one. One of the most popular TSL for the extrinsic approach was proposed by Camacho and Ortiz [7] in 2D (and Pandolfi and Ortiz [33] in 3D). There, the cohesive law is a linear decreasing function of the effective opening displacement and is derived from a free potential energy. 
In this work we have used a TSL, previously reported in [45], which allows us to define two separate values for energy dissipation in mode I and II $\left(G_{c, I}\right.$ and $\left.G_{c, I I}\right)$. The law has been developed starting from the classical model of Camacho and Ortiz but relaxing the hypothesis of a well-defined energy potential (as previously done in [5] but for the intrinsic cohesive model of Xu and Needleman [51]). The tractions are assumed to be a function of an effective scalar displacement, which has the following form:

$$
\delta=\sqrt{\frac{\beta^{2}}{\kappa^{2}} \Delta_{t}^{2}+\Delta_{n}^{2}}
$$

where $\Delta_{n}$ and $\Delta_{t}$ represent the normal and the tangential separation over the cohesive surface with unit outward normal $\mathbf{n}$ and unit tangential vector $\mathbf{t}$ respectively. The parameter $\beta$ accounts for the coupling between normal and tangential displacement, whereas $\kappa$ enables us to define the ratio between the dissipated fracture energy in mode II $\left(G_{c, I I}\right)$ and in mode I $\left(G_{c, I}\right)$

$$
\kappa=\frac{G_{c, I I}}{G_{c, I}}
$$

In case of crack opening the traction vector is defined as follows:

$$
\mathbf{T}=\left(\frac{\beta^{2}}{\kappa} \Delta_{t} \mathbf{t}+\Delta_{n} \mathbf{n}\right) \frac{\sigma_{c}}{\delta}\left(1-\frac{\delta}{\delta_{c}}\right) \quad \text { for } \delta=\delta_{\max }
$$

where $\sigma_{c}$ represents the local material strength and $\delta_{c}$ denotes the effective relative displacement beyond which complete decohesion occurs, while $\delta_{\max }$ stores the maximal effective opening displacement attained and enables thus to account for the irreversibility of the law (similarly to [7]). Unloading or reloading occurs if $\delta<\delta_{\max }$, which results in the following tractions:

$$
\mathbf{T}=\left(\frac{\beta^{2}}{\kappa} \Delta_{t} \mathbf{t}+\Delta_{n} \mathbf{n}\right) \frac{\sigma_{c}}{\delta_{\max }}\left(1-\frac{\delta_{\max }}{\delta_{c}}\right) \quad \text { for } \delta<\delta_{\max }
$$

\subsection{Contact enforcement}

Since the failure process of concrete can involve, besides cracking, frictional contact between the cracked rough surfaces, one has to enforce the impenetrability condition. For this purpose it is possible to simply avoid contact by adding a penalty term in case of negative normal opening ( $\Delta_{n}<0$ in the TSL). However, because our goal is to be able to deal with numerous asperities entering into contact and large displacements, we have preferred to couple the TSL with a contact algorithm. Since our numerical setup is implemented in an explicit dynamic code, we have selected an explicit master-slave contact algorithm called decomposition contact response (DCR) developed by Cirak and West [11]. This method resorts to the conservation of linear and angular momentum, while the impenetrability condition is enforced directly on the displacements, e.g. by projecting the impacting slave nodes on the penetrated master surface (Fig. 11. The quantity of motion of the contacting nodes after collision is 


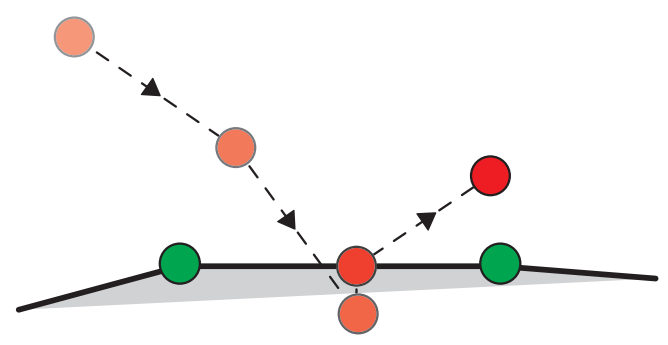

Fig. 1: Penetration removed by projecting back penetrating node on the master surface

governed by the following equations

$$
\begin{aligned}
\mathbf{p}_{i}^{t_{i}^{+}}-\mathbf{p}^{t_{i}^{-}} & =\lambda \nabla_{\mathbf{x}} g\left(\mathbf{x}^{t_{i}^{+}}\right) \\
{\left[\mathbf{p}^{T} \mathbf{M}^{-1} \mathbf{p}\right]_{t_{i}^{-}}^{t_{i}^{+}} } & =0
\end{aligned}
$$

where $\mathbf{p}=\mathbf{M} \dot{\mathbf{x}}$ represents the momentum vector of slave and master nodes ( $\mathbf{x}$ position vector), $g$ is the gap function, $\lambda$ a scalar parameter and $t_{i}^{-}$and $t_{i}^{+}$refer to the stage before and after projection within the same time step. According to these equations the post-impact velocities of the contacting nodes need to be corrected as follows:

$$
\dot{\mathbf{x}}^{+}=\dot{\mathbf{x}}^{-}-\dot{\mathbf{x}}_{n}^{-}\left(1+c_{\text {res }}\right)-\dot{\mathbf{x}}_{\text {fric }}^{-}
$$

where $c_{\text {res }}$ represents the coefficient of restitution, which can range between zero (completely inelastic contact) and one (perfectly elastic contact) and the superscripts ${ }^{+}$and ${ }^{-}$denote quantities before and after projection respectively. For the remainder of the paper we will keep $c_{\text {res }}$ equal to zero. $\dot{\mathbf{x}}_{\text {fric }}$ accounts for friction, while $\dot{\mathbf{x}}_{n}$ represents the normal quantity of motion exchanged during impact and is defined by

$$
\dot{\mathbf{x}}_{n}=\left(\frac{(\nabla g)^{T} \dot{\mathbf{x}}}{(\nabla g)^{T} \mathbf{M}^{-1} \nabla g}\right) \mathbf{M}^{-1} \nabla g
$$

In order to account for friction the relative motion between the contacting triplets (two master nodes and one slave node) needs to be corrected according to a simple Coulomb friction law which accounts for stick/slip. To this end one needs to compute the slide components of the velocity, which are given by

$$
\dot{\mathbf{x}}_{\text {slide }}=\mathbf{M}^{-1}(\nabla \mathbf{h})^{T}\left(\frac{(\nabla \mathbf{h}) \dot{\mathbf{x}}}{(\nabla \mathbf{h}) \mathbf{M}^{-1}(\nabla \mathbf{h})^{T}}\right)-\dot{\mathbf{x}}_{n}
$$

where $\mathbf{h}$ is the separation vector between impacting node and target segment. $\dot{\mathbf{x}}_{\text {slide }}$ represents therefore the velocity leading to a tangential relative motion between the bodies and corresponds to the maximal impulse which can be delivered during friction (i.e. stick case). Whereas, in case of slip, according to Coulomb's friction law, the 
correction depends on the coefficient of friction $\mu$ and on the amount of exchanged motion during impact $\dot{\mathbf{x}}_{n}$.

$$
\dot{\mathbf{x}}_{\mu}=\mu \frac{\left\|\dot{\mathbf{x}}_{n}^{T} \mathbf{M}^{-1} \dot{\mathbf{x}}_{n}\right\|}{\left\|\dot{\mathbf{x}}_{\text {slide }}^{T} \mathbf{M}^{-1} \dot{\mathbf{x}}_{\text {slide }}\right\|} \dot{\mathbf{x}}_{\text {slide }}
$$

Thus, the delivered frictional impulse will be equal to the smallest value between the slip (12) and stick (11) velocity:

$$
\dot{\mathbf{x}}_{\text {fric }}=\min \left(\dot{\mathbf{x}}_{\text {slide }}, \dot{\mathbf{x}}_{\mu}\right)
$$

\subsection{Coupling}

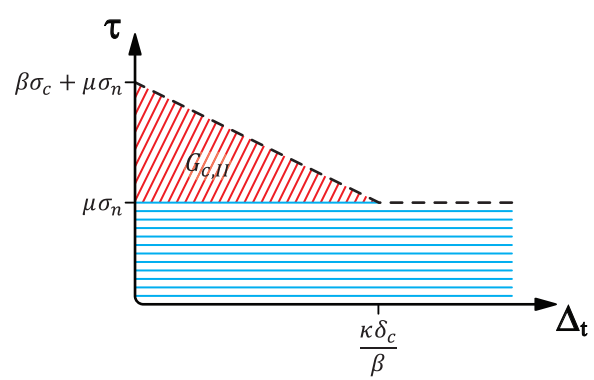

(a)

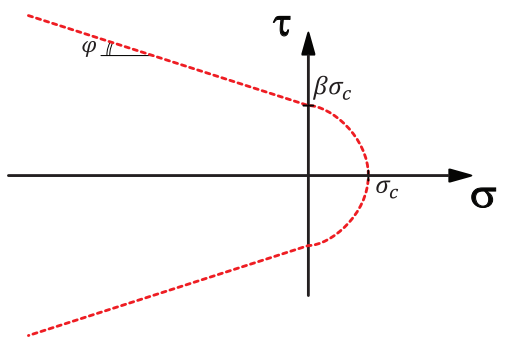

(b)

Fig. 2: (a) Shear stress-tangential opening displacement relationship for a growing crack in mode II and (b) strength failure envelope

The TSL and the contact algorithm are coupled together. I.e. if a crack is growing under compression we consider the onset of fracture to start in conjunction with the onset of friction. This is illustrated in Figure 2a where for a growing crack in Mode II the shear stress has to exceed $\beta \sigma_{c}+\mu \sigma_{n}$ in order to activate the cohesive zone. At the end of the decohesion process (when $\Delta_{t}$ reaches $\kappa \delta_{c} / \beta$ ) a fracture energy corresponding to $G_{c, I I}$ multiplied by the length of the cohesive zone will have been dissipated and the zone will undergo a continuous transition from debonding to pure frictional sliding. This approach produces a strength failure envelope for concrete drawn in Figure $2 \mathrm{~b}$ Whenever the inter-element stress between two elements reaches a point on the dotted line a cohesive element has to be inserted. The parameters $\left(\sigma_{c}, \delta_{c}, \mu, \kappa\right.$ and $\beta$ ) of the interfacial zone need to be identified according to the chosen material as reported in Section 3 


\section{Meso-scale model of concrete}

Concrete is a heterogeneous brittle material made of various components, which are present in different proportions. This produces a quasi-brittle material, whose mechanical behavior is defined by the wide range of the ingredients in the mixture. Considering concrete at a meso-scale level of observation allows to describe it as a biphasic material: aggregates embedded in a mortar paste matrix. In our model only medium and large aggregates are represented explicitly. Whereas, small aggregates and other components are assumed to be mixed up with the cement paste establishing the matrix phase. Besides those two components, the interfaces between the two constituents, called interfacial transition zone (ITZ), are represented by dynamically inserted elements with the presented cohesive-frictional capability.

\subsection{Mesh generation and aggregate distribution}

The specimen geometry has been obtained using a random generator of irregular polygons. Inclusions with the smallest diameter ranging from $1.5 \mathrm{~mm}$ to $20 \mathrm{~mm}$ have been considered. The distribution has been chosen according to the Fuller and Thompson density curve [19] with a value for the exponent $q$ equal to 0.7 .

$$
A(d)=\left(\frac{d}{d_{\max }}\right)^{q}
$$

Where $\mathrm{A}(\mathrm{d})$ represents the percent by weight (cumulated mass under a given diameter $d$ ) and $d_{\text {max }}$ the largest aggregate in the mixture. The obtained cumulated distribution is depicted in Figure $3 \mathrm{a}$ for the generated specimen geometry of Figure $3 \mathrm{~b}$

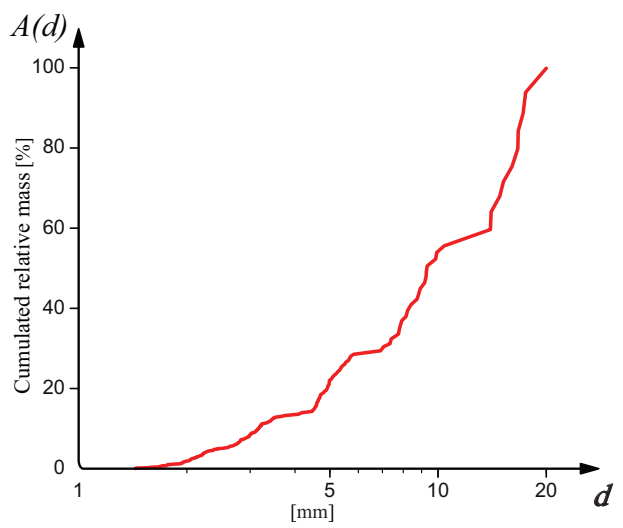

(a)

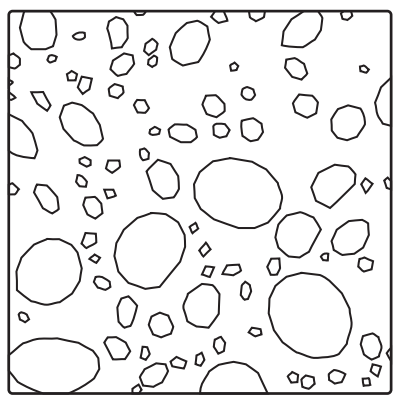

(b)

Fig. 3: (a) Computed cumulated aggregates size distribution of the concrete mesostructure (b) 
The specimen is a square with an edge size equal to $100 \mathrm{~mm}$. In order to have a regular distribution of the phases near the boundaries, the aggregates have been placed in a larger sample, from which the specimen has been cut out. The resulting ratio of aggregate area is around $31 \%$. From this geometry one can obtain meshes with different element sizes. Since a convergence study on mesh sensitivity has already been performed in previous work ([44] and [22]), we have set directly the value of the average element size to $0.5 \mathrm{~mm}$ (which gives a mesh with roughly 120000 nodes).

\subsection{Material parameters}

The meso-mechanical approach requires defining the material properties for every component. In Table 1 the material properties for the inclusions and matrix paste are summarized. Those values are generic and suitable for a usual concrete and reflect the values used (by the authors) in previous work [44].

Table 1: Material properties of the concrete's components

\begin{tabular}{lccc}
\hline Material & Density $-\rho\left[\mathrm{kg} / \mathrm{m}^{3}\right]$ & Young's modulus $-\mathrm{E}[\mathrm{GPa}]$ & Poisson's ratio $-v[-]$ \\
\hline Aggregate & 2700 & 75 & 0.2 \\
Cement paste & 2200 & 30 & 0.2 \\
\hline
\end{tabular}

The values for the three different interfaces are reported in Table 2 These material properties can be determined experimentally (for instance [39]). For this work we have chosen values of the cohesive properties (Table $3 \mathrm{a}$ similar to the ones reported in [22] while the remaining pair $(\beta, \kappa$ in Table $3 \mathrm{~b}$ ] had to be identified through a parametric study as reported in Section 4

Table 2: Cohesive properties and parameters for the interfaces (selected values for $\beta$ and $\kappa$ are shown in bold)

\begin{tabular}{lcc}
\hline Interface & Fracture Energy $-G_{c}\left(\mathrm{~J} / \mathrm{m}^{2}\right)$ & Tensile Strength $-\sigma_{c}[\mathrm{MPa}]$ \\
\hline Aggregate & 60 & 16 \\
Mortar paste & 50 & 4.7 \\
ITZ & 30 & 2.7 \\
\hline
\end{tabular}

(a)

\begin{tabular}{lccc}
\hline Interface & $\beta$ & $\kappa$ & Friction Coefficient $-\mu$ \\
\hline Aggregate & $12 \mathbf{3}$ & $12 \mathbf{3} 4$ & 0.7 \\
Mortar paste & $12 \mathbf{3}$ & $12 \mathbf{3 4}$ & 0.7 \\
ITZ & $12 \mathbf{3}$ & $12 \mathbf{3 4}$ & 0.7 \\
\hline
\end{tabular}

(b) 


\section{Uniaxial tensile/compressive loading}

In this section we analyze the dynamic tensile and compressive response of the concrete model.

\subsection{Initial and boundary conditions}

The samples are loaded under displacement control with an imposed strain rate $\dot{\varepsilon}$. For tension all the nodes of the finite element mesh which are located on the upper (respectively lower) boundary are forced to move at a constant velocity $V_{0 y}=V_{0}$ (respectively $V_{0 y}=-V_{0}$ ) as illustrated in Fig.4a):

$$
V_{0}=\dot{\varepsilon} \frac{h}{2}
$$

where $h$ is the height of the studied specimen. To avoid important stress wave propagation and an early fracture near the boundaries [30], all nodes of the finite-element mesh are prescribed an initial velocity as illustrated in Fig.4a

$$
V_{y}(y)=\frac{2 V_{0}}{h} y
$$

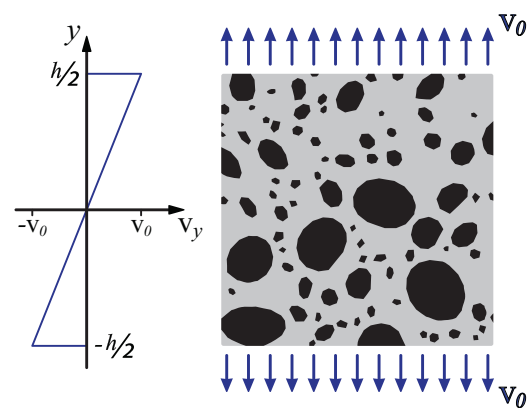

(a)

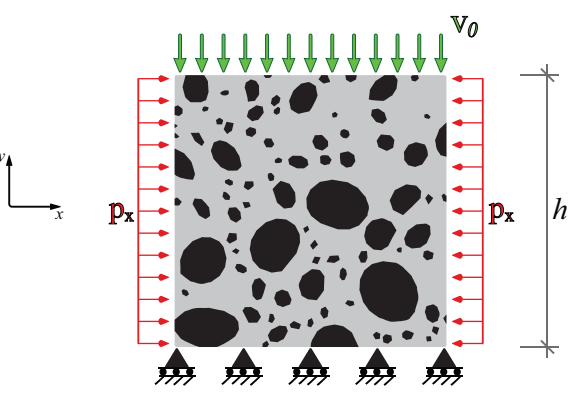

(b)

Fig. 4: Boundary and initial conditions for specimen loaded in (a) tension and (b) compression

In case of compression (Fig. 4b nodes located at the upper edge of the finite element mesh are forced to move at a certain constant velocity $V_{0}$ :

$$
V_{0}=-\dot{\varepsilon} h
$$

Whereas, the lower boundary is supported in y-direction and therefore the motion of the nodes belonging to this edge is blocked in the vertical direction. If lateral confinement $\left(p_{x}\right)$ is applied (Section 5], the sample is first loaded statically with a hydrostatic 
pressure corresponding to $p_{x}$. After this step, the dynamic loading is applied until the end of the simulation.

To obtain the stress-strain curves presented thereafter, we define the macroscopic stress $(\sigma)$ as the boundary reaction force $F_{y}$ divided by the initial width, and the macroscopic strain $(\varepsilon)$ as the change in height divided by the initial height $h$. In case of compression, the compressive stress and compressive strain are identified with $\sigma_{c}$ and $\varepsilon_{c}$ respectively. Moreover, during simulation, a slight material damping has been adopted in order to compensate the slight increase of internal energy (due to the enforcement of the impenetrability through projection [?]) and reduce numerical oscillations.

\subsection{Identification of model's interface parameters through simulations}

In order to identify the two remaining parameters of the cohesive law, $\beta$ and $\kappa$, we have ran some simulations in order to extract them indirectly by comparing the macroscopic stress-strain behavior with concrete experimental behavior.

Since these two parameters influence mode II cracking, and have therefore little influence on the peak tensile strength and more generally on the global macroscopic behavior of the specimens subjected to uniaxial tension, the fitting has been conducted by examining the response in unconfined compression for a loading rate $\dot{\varepsilon}=1 \mathrm{~s}^{-1}$. The influence of $\beta$ has been investigated first. Its value affects the shear strength of the interfaces, which changes considerably the compressive peak strength of concrete as depicted in Fig. 5a With an increasing value of $\beta$, one obtains a higher compressive strength. Since with the interface properties of Table 3 a a tensile strength slightly

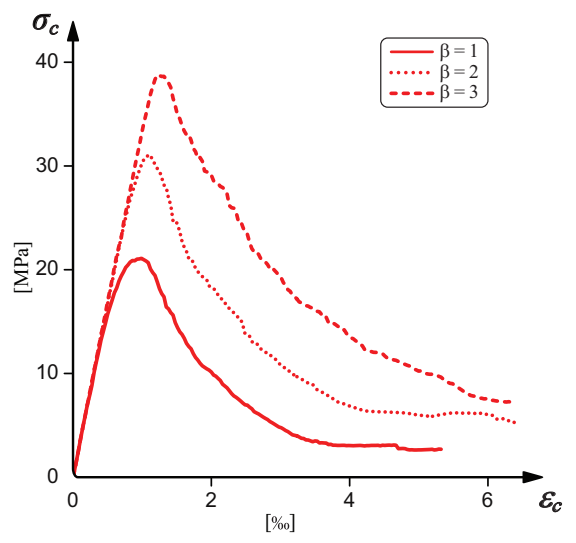

(a)

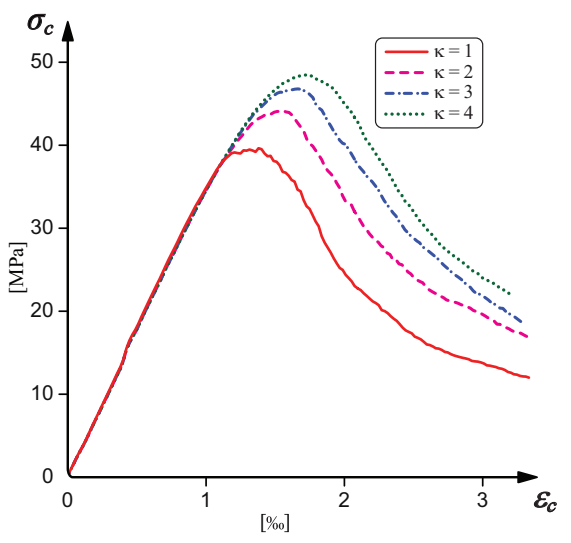

(b)

Fig. 5: (a) Influence of the $\beta$ parameter on the compressive stress-strain behavior of concrete (for $\kappa=1$ ) and (b) influence of $\kappa$ (with $\beta=3$ ) for $\dot{\varepsilon}=1 s^{-1}$

lower than $4 \mathrm{MPa}$ is obtained, the authors have decided to set the value of $\beta$ equal to 
three (a lower value compared to the one chosen in [41]). This leads to a compressive strength roughly one order of magnitude higher than the tensile one, which seems a usual ratio for a conventional concrete.

The stress-strain behavior is also affected by $\kappa$, which increases primarily the dissipated fracture energy and therefore modifies mostly the post-peak behavior and shifts the transition to softening towards higher strain values. This trend is graphically illustrated in Fig. 5b. Note that an increasing value of $\kappa$ increases also the peak strength, but not as much as with $\beta$. Therefore, in order to obtain a concrete with softening starting around $\varepsilon_{c}=0.002$, we decided to fix the value of $\kappa$ at three. This results in a concrete with a compressive strength of roughly $46 \mathrm{MPa}$ at a compressive peak strain around 0.0018 . The stress-strain behavior is depicted for tension and compression in Fig. It is clear that the tension/compression asymmetry is well captured.

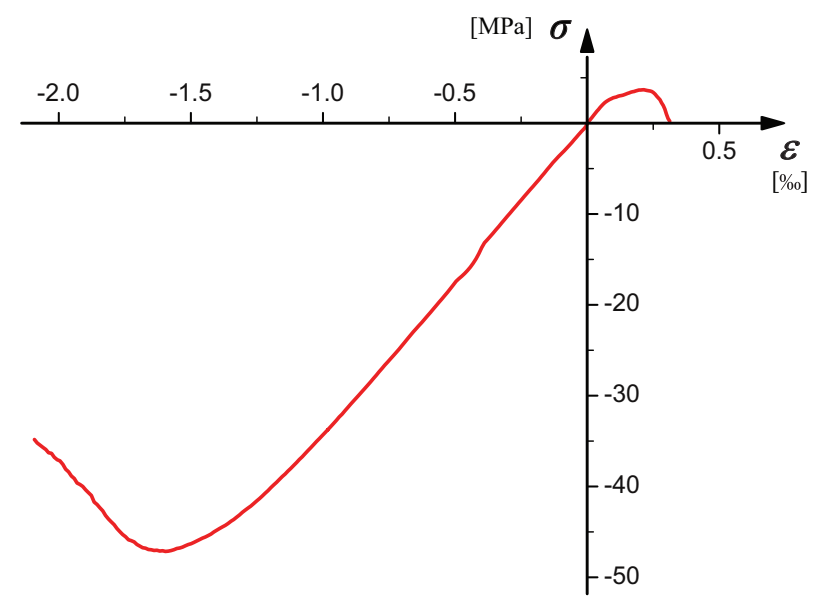

Fig. 6: Dissymmmetric tensile/compressive behavior of concrete $\left(\dot{\varepsilon}=1 s^{-1}\right)$

\subsection{Rate effect}

In this subsection we analyze the behavior of concrete subjected to tension and unconfined compression under different loading rates.

\subsubsection{Tensile response}

It is well-known that experimental results on dynamic tension tests show a rate sensitivity of the tensile strength [46 18]. In quasi-statics, the macroscopic tensile strength is mainly governed in our case by the Interfacial Transition Zone strength and toughness (ITZ between the aggregates and the mortar paste) and not by the meso-structure [35 22]. For low strain rates $-\dot{\varepsilon}<1 / \mathrm{s}-$ the dynamic resistance increase is mainly 
due to the presence of water in the material [40] and we have a slight Dynamic Increase Factor (DIF) - equal to the ratio of static versus dynamic strengths. For higher strain rates $-\dot{\varepsilon}>1 / \mathrm{s}$ - the usual explanation of a more important DIF is the transition between single cracking in quasi-statics to diffuse cracks in dynamics.

The results of the strain-stress curves obtained for our numerical simulations in tension for several strain rates are presented in Fig. 7 As expected, the transition from a unique crack to diffuse cracking has a strong influence on the macroscopic behavior of concrete. Increasing the strain rate delays (relatively) the coalescence of the microcracks, which rises the peak strength. We can notice that in our case the dynamic increase factor is equal to almost 2 for $\dot{\varepsilon}=100 / \mathrm{s}$ which can be too slight compared to experimentally reported values $(\sim 3[49])$. This implies that we may have to take into account rate dependency at the material level, for example by linking the cohesive strength $\sigma_{c}$ to the rate of deformation of the surrounding material and to the crack opening rate, in order to achieve better agreement with experimental results. We may nonetheless conclude that the dynamic increase factor is mostly due to a "structural effect", with probably a small part due to a viscous behavior of the matrix paste.

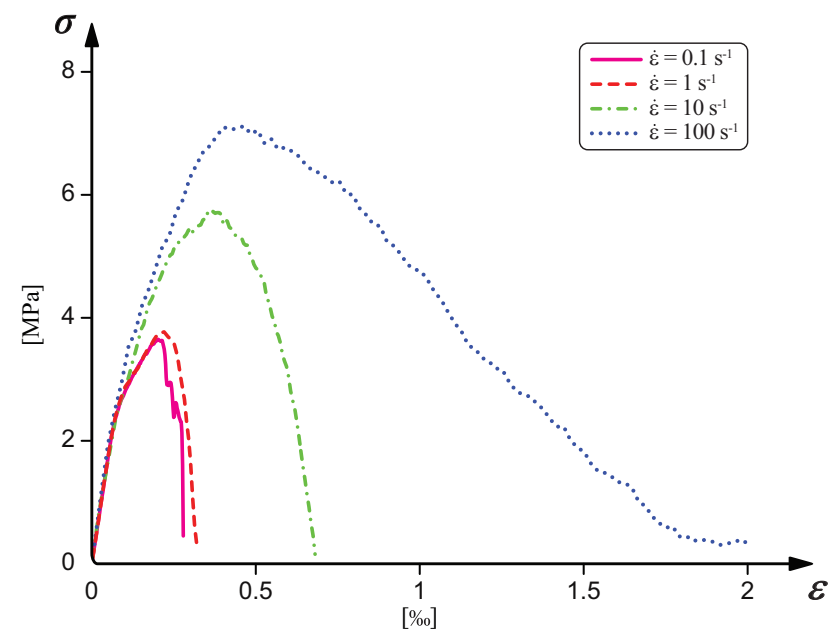

Fig. 7: Influence of strain rate on the tensile stress-strain curves

Fig. 8 shows the final crack patterns for a low and a high strain rate. For $\dot{\varepsilon}=0.1 / \mathrm{s}$ we have a unique macroscopic crack, while for $\dot{\varepsilon}=100$ /s we obtain diffuse cracks. In both cases, microcracks, for the most part, succeed to find paths around the aggregates.

Fig. 9 depicts the evolution of the dissipated fracture energy as a function of the macroscopic strain of the specimen for different loading rates. One can remark from this figure that the dissipated fracture energy strongly depends on the loading rate even with a rate independent local fracture energy. Nevertheless as for the strength, the DIF for the dissipated fracture energy $\left(W_{G}\right)$ in the specimen is slightly lower than 




(a)

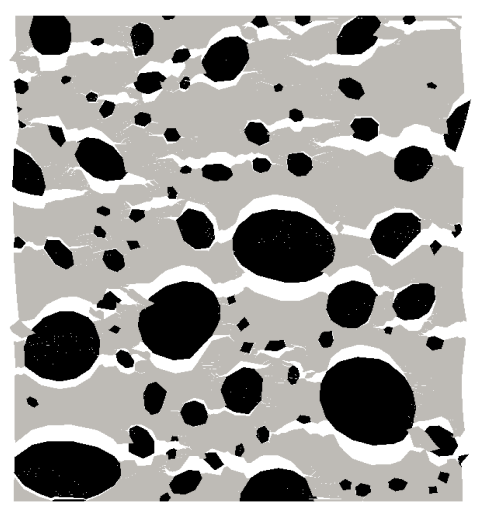

(b)

Fig. 8: Influence of strain rate on the crack path in uniaxial tension: (a) $\dot{\varepsilon}=0.1 s^{-1}$, (b) $\dot{\varepsilon}=100 s^{-1}$. Displacement has been magnified by a factor of 100 .

the experimental one [49]. For more detail on the tensile response of our model, one can refer to [22].

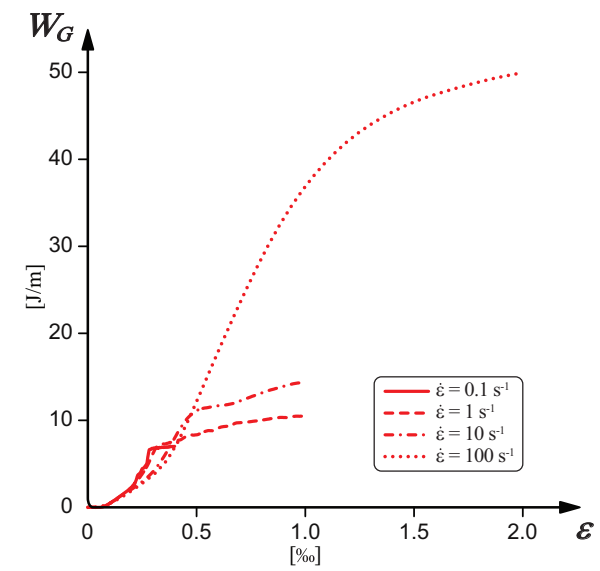

Fig. 9: Influence of strain rate on the dissipated energy for tensile loading

\subsection{2 compressive response}

As for tension, experimental results [3] show a clear rate sensitivity under compressive loading. Commonly, the stress increase can be explained with lateral inertial 
confinement (Poisson's effect) and a more diffuse micro-cracking beside eventual material rate hardening mechanisms.

Fig. 10] shows the computed stress-strain curve for different loading rates. The results display a strain rate hardening with a DIF of about two for a strain rate of $\dot{\varepsilon}=100$ $s^{-1}$. In contrast to our computed tensile DIF, this increase factor is consistent with experimental results [3]. This results highlights the strong effect of lateral inertial confinement. Indeed, in our simulations, material we do not consider any rate effect at the material level.



Fig. 10: Stress-strain response for different strain rates under compressive loading

Fig. 11 shows the crack pattern for $\dot{\varepsilon}=1 s^{-1}$ and $100 s^{-1}$. Cracks tend to propagate within the matrix phase bypassing the inclusions except for few big aggregates that have been crossed. One can notice that due to the absence of a horizontal constraint at the upper and lower boundaries of the specimen, the crack pattern does not show the formation of a characteristic cone, as mostly observed during simple compression experiments. Moreover, the cracks are aligned parallel to the loading direction, conversely to a perpendicular orientation in case of tension as shown in Fig. 8

The evolution of the dissipated fracture energy $\left(W_{G}\right)$ and frictional work $\left(W_{\mu}\right)$ in the specimen is depicted in Figs. $12 \mathrm{a}$ and $12 \mathrm{~b}$ respectively. Both figures show that the raising number of cracks with increasing strain rate causes a higher dissipation of energy within the damaged specimen. This mechanism leads to a higher compressive strength and larger area (thus toughness) under the stress-strain curve. In particular, the post-peak dissipation of energy seems to be affected more by friction than by dissipation of fracture energy. Therefore, the gain in strength can be traced back to an inertial effect alone (as suggested for instance in [12]). 


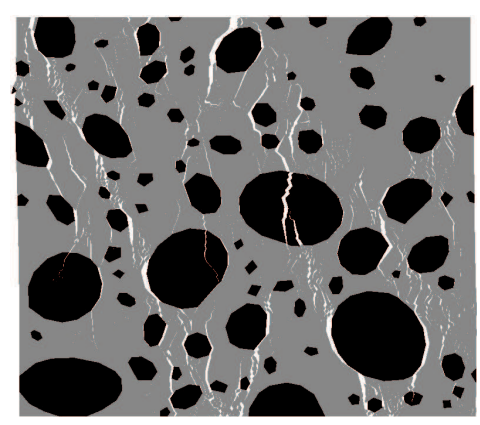

(a)

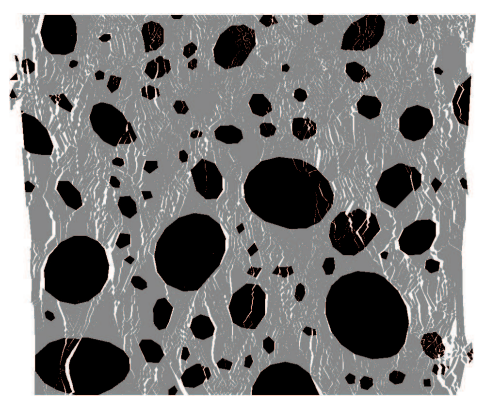

(b)

Fig. 11: Influence of strain rate on the crack path in compression: (a) $\dot{\varepsilon}=1 s^{-1}$, (b) $\dot{\varepsilon}$ $=100 s^{-1}$. Displacement has been magnified by a factor of 5 (a) and 3 (b).

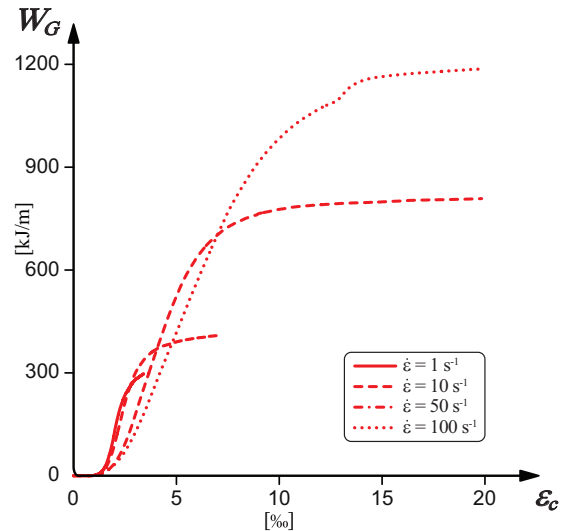

(a)

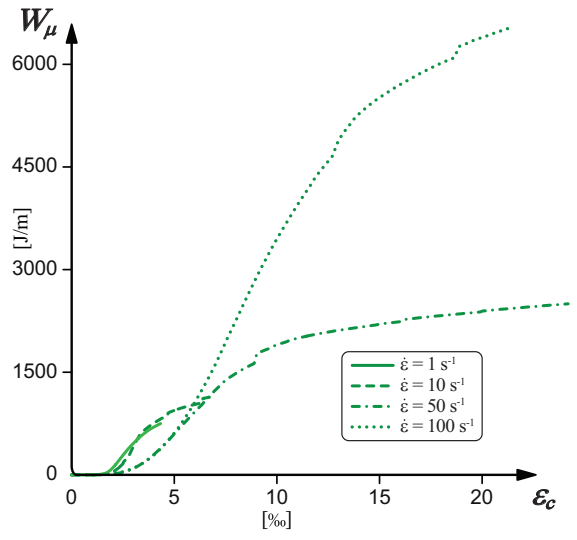

(b)

Fig. 12: (a) Dissipated fracture energy and (b) dissipated frictional work for different loading rates

\section{Influence of lateral confinement}

We now investigate the behavior of concrete subjected to a small lateral confinement under a strain rate $\dot{\varepsilon}=1 s^{-1}$. The concrete specimens have been subjected to three level of transversal confinement pressures $\left(p_{x}\right): 0,3$ and $6 \mathrm{MPa}$.

Figure 13 shows that the confining stress increases substantially the compressive strength as well as the longitudinal compressive peak strain. Moreover, one can notice that confining concrete results in a decrease of the slope of the post-peak branch indicating therewith a moderate rise in the ductility of concrete. From those results one can compute the gain in concrete strength of the confined specimens. Usually, in concrete research, the Mohr-Coulomb failure criterion can be assumed to describe 


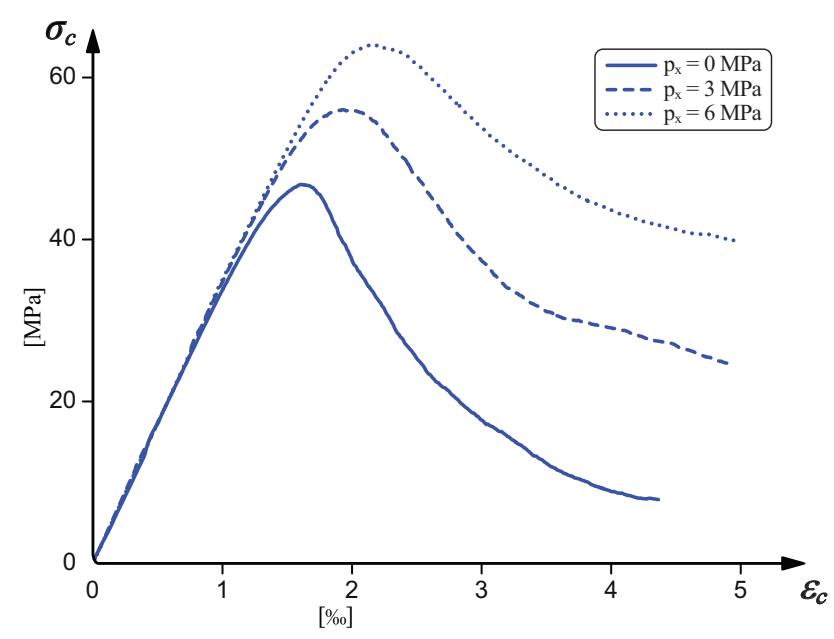

Fig. 13: Influence of confining pressure on the stress-strain behavior $\left(\dot{\varepsilon}=1 s^{-1}\right)$

the sliding failure in a confined concrete. This can be expressed as follows

$$
\sigma_{c}=f_{c}+k * p_{x}
$$

where $f_{c}$ represents the unconfined compressive strength of concrete and $k$ is a constant, which is usually set to four [37 28 8] for triaxial tests. In our case, we obtain a $k$ somewhat lower with a value of roughly three. A better modeling could perhaps be obtained by increasing the value of the friction coefficient and the pair $(\beta, \kappa)$. The comparison between the dissipated energies is illustrated in Figure 14 From Figure 14a one can remark that an increase in the lateral confinement will delay the opening of cracks and thereafter the start of the dissipation of fracture energy. This phenomenon has already been observed with a mesoscale modeling of ceramics 48, 27]. However, the rise in the horizontal pressure ultimately increases the amount of dissipated fracture energy. This is because cracks are more prone to open following a mode II fashion, which is bounded with a larger value of stored fracture energy than mode I, and also because there is a more diffuse crack network. Fig. 15 shows this crack network. It also illustrates that applying a confinement pressure forces the cracks to propagate at a faulting angle of roughly $30^{\circ}$, whereas their paths were more vertical for unconstrained compression (Fig. [11a). The increase in the faulting angle orientation, from axial splitting for unconfined concrete to shear faulting for specimens subjected to lateral confinement, appears to be in agreement with analytical models (see for instance [25] for rock mechanics).

Since we are using an explicit representation of cracks, it is possible to monitor their time evolution and to extract relevant information. We have chosen to follow the formation of the longest crack cluster (which is a group of fully broken interfaces which are interconnected to each other), $L_{c, \max }$, as well as the total number of crack clusters $N B_{c}$ (as was done for ceramics in [48 27]). The first variable has been normalized 


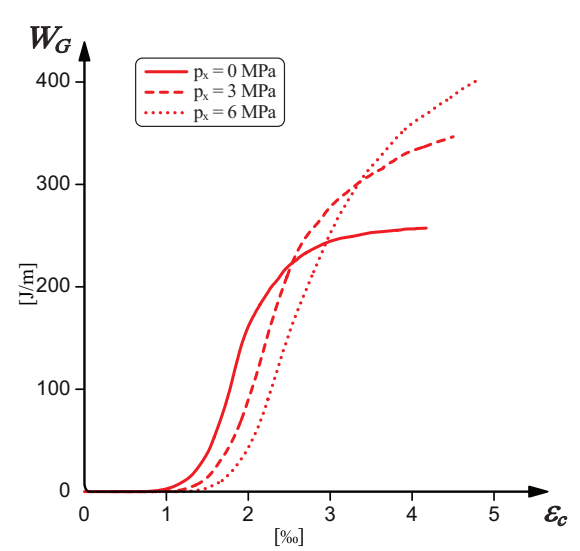

(a)

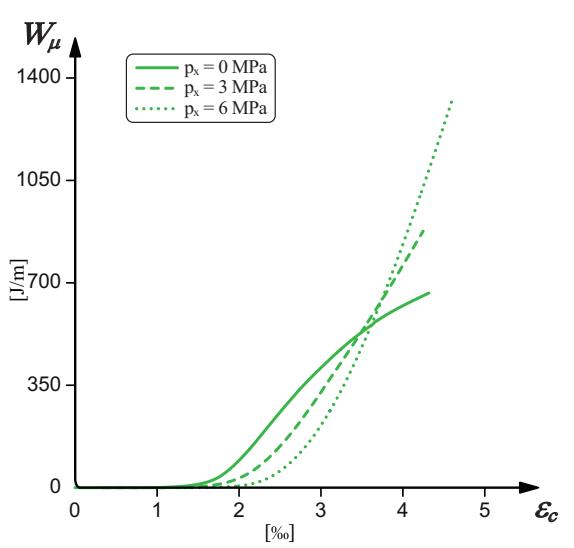

(b)

Fig. 14: (a) Dissipated fracture energy for $p_{x}=0,3$ and $6 \mathrm{MPa}$ and (b) frictional work for $p_{x}=0,3$ and $6 \mathrm{MPa}$ (for $\dot{\varepsilon}=1 s^{-1}$ )

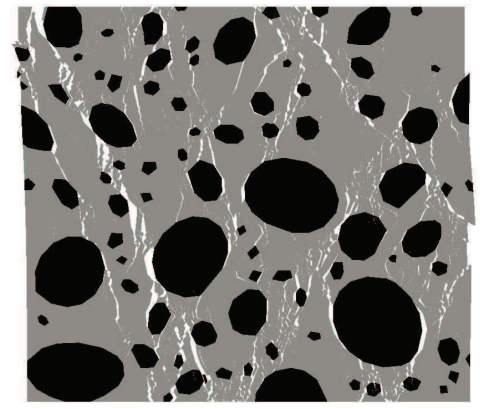

(a)

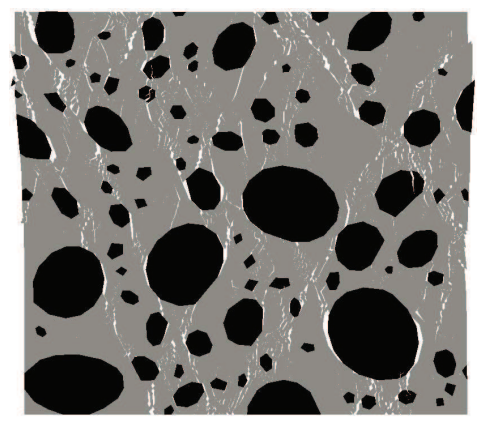

(b)

Fig. 15: Deformed specimens for confinement pressures of (a) $3 \mathrm{Mpa}$ and (b) $6 \mathrm{MPa}$. Displacement has been magnified by a factor of 5 .

with the edge size of the specimen. As already observed, an increase in the level of confinement produces a delay in the formation of the first cluster. This is noticeable from both graphs of Fig.16 Moreover, from the first graph (Fig.16a) one can see that the application of a horizontal pressure causes the longest crack to be shorter and on the same range as $h$ (unfolded length). On the other hand, the samples show a larger amount of clusters as depicted in Fig. 16b which implies that confinement delays the onset of microcracks coalescence. This can be confirmed by looking at the deformed mesh configuration. One can see from Fig. 15 that the application of the confinement pressure seems to cause a finer net of smaller cracks, which exhibit a smaller opening. Finally, by looking at Fig. 16a it appears that the softening phase can start, for the three cases considered here, when the longest cluster reaches a length of approxi- 


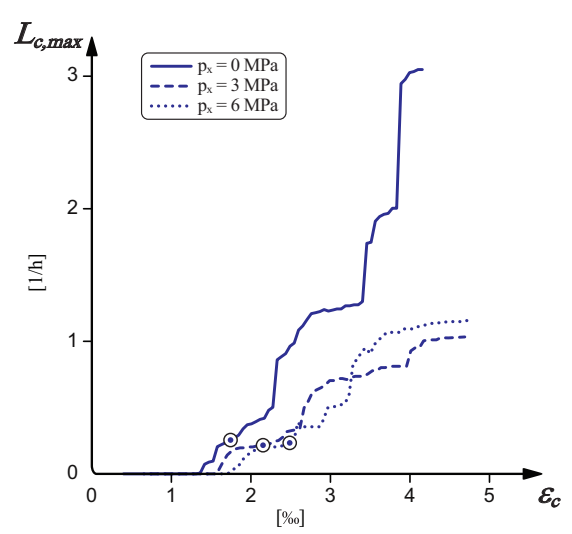

(a)

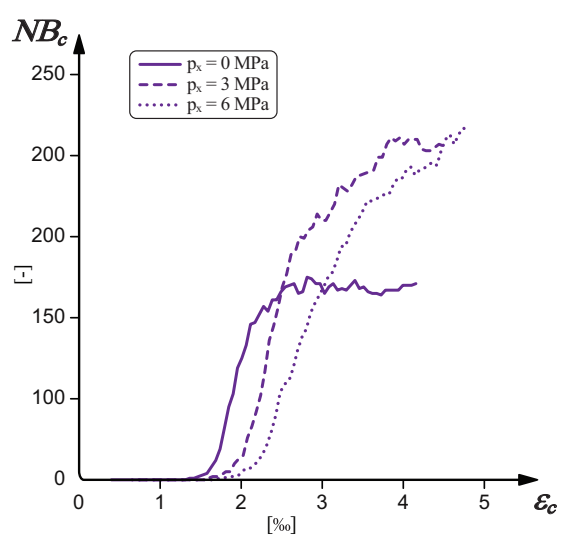

(b)

Fig. 16: (a) Maximal cluster length normalized over specimen size $h$ for $p_{x}=0,3$ and $6 \mathrm{MPa}$ and (b) number of cracks for $p_{x}=0,3$ and $6 \mathrm{MPa}$. The length at which softening starts, represented by circles on graph (a), does not depend on the amount of confinement.

mately $0.2-0.25 h$ (circles on the strain- $L_{c, \max }$ curve indicate the strain immediately after peak strength has been reached) and thus does not depend on the level of applied lateral pressure.

One can notice that the simulated behavior shows a quite brittle compressive softening despite the applied horizontal pressure (in particular at the early stage of softening). One reason could be traced back to the chosen coupling between friction and debonding, which produces an increase in the peak strength but leads to an initial larger negative slope during the softening phase. Other possibilities could reside in an insufficient dissipation of frictional energy and in the choice of the parameters $\beta$ and $\kappa$.

\section{Discussion and Conclusions}

In this paper we have presented a 2D dynamical meso-mechanical model of concrete with cohesive/frictional capability for transient dynamics. The meso-scale approach enables us to represent aggregates and mortar explicitly, thus allowing all material parameters to be physically identified. Both continuum phases are considered to behave elastically while initiation, coalescence and propagation of cracks are modeled by dynamically inserted interface elements with the proposed cohesive frictional capability. The debonding process is controlled by a novel extrinsic traction separation law which accounts for path dependent behavior and therefore enables us to define two separate values for energy dissipation in mode I and II. The impenetrability condition is enforced directly by projecting the impacting nodes on the penetrated surface. 
We have used this model to simulate dynamic concrete's behavior in traction and compression. From the obtained results we can draw the following conclusions.

Simulations in tension as well as in compression show that the model gives an increase in peak strength and strain at failure with increasing rate of loading although the interfacial constitutive law is rate independent. This rise in strength resides in a more diffuse micro-cracking and is thereby bounded with a higher dissipation of fracture energy as well as energy dissipated through friction in case of compressive cracking. A comparison between our simulation results and experimental literature indicates that inertial forces alone in case of compressive loading are sufficient to explain the increase in strength with increasing loading rate. On the contrary, if the specimen is subjected to tensile loading, a small strain-rate dependence at the material level (material hardening) should be added in order to achieve a better agreement with experimental results.

Specimens subjected to lateral confinement in compression exhibit an increase in peak strength with increasing confining pressure. The rise in strength is consistent with experimental reported values (although somewhat lower). It has been observed that an increase in the lateral pressure produces delays in the formation of the first crack cluster and in microcracks coalescence. Dissipation of energy through fracture and friction is also an increasing function of the applied confinement. The model also shows the importance of capturing frictional mechanisms, which appear to dissipate more energy than crack-opening under compressive loading.

It is however important to emphasize that our model needs further improvement to capture better experiments. In particular, the ductility of the specimen is little affected by the confining pressure and the post-peak behavior remains fairly brittle. This could perhaps be traced back to a yet insufficient increase in the amount of dissipated frictional energy or in part to the chosen coupling between friction and the cohesion. Also the influence of internal ordering of the meso-structure has not been investigated in this paper. In the future, we plan to use our model as a basis to investigate shearing resistance (including asperities interlocking) of structural components.

Acknowledgements This material is based on the work supported by the Swiss National Foundation under Grant no. 200021 122046/1.

\section{References}

1. Barenblatt, G.I.: The mathematical theory of equilibrium of cracks in brittle fracture. Advances in Applied Mechanics 7, 55-129 (1962)

2. Bazant, Z.P., Caner, F.C., Adley, M.D., Akers, S.A.: Fracturing rate effect and creep in microplane model for dynamics. Journal of Engineering Mechanics-ASCE 126(9), 962-970 (2000)

3. Bischoff, P.H., Perry, S.H.: Compressive behaviour of concrete at high strain rates. Materials and Structures 24, 425-450 (1991)

4. Bolander Jr., J.E., Saito, S.: Fracture analysis using spring networks with random geometry. Engineering Fracture Mechanics 61(5-6), 569-591 (1998)

5. Von den Bosch, M.J., G., S.P.J., Geers, M.G.D.: A critical evaluation of the exponential Xu and Needleman cohesive zone law for mixed mode decohesion. Engineering Fracture Mechanics 72, 2247-2267 (2005)

6. Caballero, A., López, C.M., Carol, I.: 3D meso-structural analysis of concrete specimens under uniaxial tension. Computer Methods in Applied Mechanics and Engineering 195(52), 7182-7195 (2006) 
7. Camacho, G.T., Ortiz, M.: Computational modelling of impact damage in brittle materials. International Journal of Solids and Structures 33(20-22), 2899-2938 (1996)

8. Candappa, D.C., Sanjayan, J.G., Setunge, S.: Complete Triaxial Stress-Strain Curves of High-Strength Concrete. Journal of Materials in Civil Engineering 13(3), 209-215 (2001)

9. Carol, I., López, C.M., Roa, O.: Micromechanical analysis of quasi-brittle materials using fracturebased interface elements. International Journal for Numerical Methods in Engineering 52(1-2), 193 215 (2001)

10. Cervenka, J., Chandra Kishen, J.M., Saouma, V.E.: Mixed mode fracture of cementitious bimaterial interfaces; part ii: Numerical simulation. Engineering Fracture Mechanics 60(1), 95-107 (1998)

11. Cirak, F., West, M.: Decomposition contact response (DCR) for explicit finite element dynamics. International Journal for Numerical Methods in Engineering 64, 1078-1110 (2005)

12. Cotsovos, D.M., Pavlović, M.N.: Numerical investigation of concrete subjected to compressive impact loading. Part 1: A fundamental explanation for the apparent strength gain at high loading rates. Computers and Structures 86, 145-163 (2008)

13. Cusatis, G.: Strain-rate effects on concrete behavior. International Journal of Impact Engineering 38(4), 162-170 (2011)

14. Desmorat, R., Chambart, M., Gatuingt, F., Guilbaud, D.: Delay-active damage versus non-local enhancement for anisotropic damage dynamics computations with alternated loading. Engineering Fracture Mechanics 77(12), 2294-2315 (2010)

15. Desmorat, R., Gatuingt, F., Ragueneau, F.: Nonstandard Thermodynamics Framework for Robust Computations with Induced Anisotropic Damage. International Journal of Damage Mechanics 19(1), 53-73 (2010)

16. Dubé, J.F., Pijaudier-Cabot, G., La Borderie, C.: Rate dependent damage model for concrete in dynamics. Journal of Engineering Mechanics-ASCE 122(10), 939-947 (1996)

17. Dugdale, D.S.: Yielding of steel sheets containing slits. Journal of the Mechanics and Physics of Solids 8, 100-108 (1960)

18. Erzar, B., Forquin, P.: An Experimental Method to Determine the Tensile Strength of Concrete at High Rates of Strain. Experimental Mechanics 50(7), 941-955 (2010)

19. Fuller, W.B., Thompson, S.E.: The Laws of Proportioning Concrete. Journal of Transportation Division 59 (1907)

20. Gatuingt, F., Desmorat, R., Chambart, M., Combescure, D., Guilbaud, D.: Anisotropic 3D delaydamage model to simulate concrete structures. Revue Européenne de mécanique numérique 17, 74060 (2008)

21. Gatuingt, F., Pijaudier-Cabot, G.: Coupled damage and plasticity modeling in transient dynamic analysis of concrete. International Journal for Numerical and Analytical Methods in Geomechanics 26, 1-24 (2002)

22. Gatuingt, F., Snozzi, L., Molinari, J.F.: Numerical determination of the tensile response and the dissipated fracture energy of concrete: role of the meso-structure and influence of the loading rate. International Journal for Numerical and Analytical Methods in Geomechanics, accepted

23. Grassl, P., Jirásek, M.: Meso-scale approach to modelling the fracture process zone of concrete subjected to uniaxial tension. International Journal of Solids and Structures 47(7-8), 957-968 (2010)

24. Grassl, P., Rempling, R.: A damage-plasticity interface approach to the meso-scale modelling of concrete subjected to cyclic compressive loading. Engineering Fracture Mechanics 75(16), 4804-4818 (2008)

25. Horii, H., Nematnasser, S.: Compression-Induced Microcrack Growth in Brittle Solids: Axial Splitting and Shear Failure. Journal of Geophysical Research 90, 3105-3125 (1985)

26. Kozicki, J., Tejchman, J.: Effect of aggregate structure on fracture process in concrete using 2 d lattice model. Archives of Mechanics 59, 365-384 (2007)

27. Kraft, R.H., Molinari, J.F., Ramesh, K.T., Warner, D.H.: Computational micromechanics of dynamic compressive loading of a brittle polycrystalline material using a distribution of grain boundary properties. Journal of the Mechanics and Physics of Solids 56 56(8), 2618-2641 (2008)

28. Lahlou, K., Aitcin, P., Chaallal, O.: Behaviour of high-strength concrete under confined stresses. Cement and Concrete Composites 14(3), 185-193 (1992)

29. López, C.: Microstructural analysis of concrete fracture using interface elements. Application to various concretes. Ph.D. thesis, Universitat Politcnica de Catalunya, Spain (1999)

30. Miller, O., Freund, L.B., Needleman, A.: Modeling and simulation of dynamic fragmentation in brittle materials. International Journal of Fracture 96(2), 101-125 (1999)

31. Newmark, N.M.: A method of computation for structural dynamics. Journal of Engineering Mechanics 85, 67-94 (1959) 
32. Ottosen, N.S.: Constitutive model for short-time loading of concrete. Journal of the Engineering Mechanics Division-ASCE 105(1), 127-141 (1979)

33. Pandolfi, A., Ortiz, M.: Finite-deformation irreversible cohesive elements for three dimensional crackpropagation analysis. International Journal for Numerical Methods in Engineering 74, 1393-1420 (1999)

34. Pedersen, R.R., Simone, A., Sluys, L.J.: An analysis of dynamic fracture in concrete with a continuum visco-elastic visco-plastic damage model. Engineering Fracture Mechanics 75, 3782-805 (2008)

35. Pedersen, R.R., Simone, A., Stroeven, M., Sluys, L.J.: Mesoscopic modelling of concrete under impact. In: 6th International Conference on Fracture Mechanics of Concrete and Concrete Structures FRAMCOS VI, pp. 571-578 (2007)

36. Pontiroli, C., Rouquand, A., Mazars, J.: Predicting concrete behaviour from quasi-static loading to hypervelocity impact An overview of the PRM model. European Journal of Environmental and Civil Engineering 14(6-7), 703-727 (2010)

37. Richart, F.E., Brandtzaeg, A., Brown, R.L.: Failure of Plain and Spirally Reinforced Concrete in Compression. Tech. Rep. 190, University of Illinois, Urbana (1929)

38. Roelfstra, P., Sadouki, H., Wittmann, F.: Le béton numerique. Materials \& Structures - RILEM 18, 309-317 (1985)

39. Rosselló, C., Elices, M., Guinea, G.V.: Fracture of model concrete: 2. Fracture energy and characteristic length. Cement and Concrete Research 36, 1345-1353 (2006)

40. Rossi, P., van Mier, J.G.M., Boulay, C., Le Maou, F.: The dynamic behaviour of concrete: influence of free water. Materials and Structures 25, 509-514 (1992)

41. Ruiz, G., Ortiz, M., Pandolfi, A.: Three-dimensional finite-element simulation of the dynamic Brazilian tests on concrete cylinders. International Journal for Numerical Methods in Engineering 48(7), 963-994 (2000)

42. Schlangen, E., van Mier, J.G.M.: Simple lattice model for numerical simulation of fracture of concrete materials and structures. Materials and Structures 25(9), 534-542 (1992)

43. Sercombe, J., Ulm, F.J., Toutlemonde, F.: Viscous hardening plasticity for concrete in high-rate dynamics. Journal of Engineering Mechanics-ASCE 124(9), 1050-1057 (1998)

44. Snozzi, L., Caballero, A., Molinari, J.F.: Influence of the meso-structure in dynamic fracture simulation of concrete under tensile loading. Cement and Concrete Research 41, 1130-1142 (2011)

45. Snozzi, L., Molinari, J.F.: A cohesive element model for mixed mode loading with frictional contact capability. International Journal for Numerical Methods in Engineering, submitted

46. Vegt, I., Pedersen, R.R., Sluys, L.J., Weerheijm, J.: Modelling of impact behaviour of concrete - An experimental approach. In: Computational Modelling of Concrete Structures - EURO-C 2006, pp. 451-458 (2006)

47. Vonk, R.A.: Micromechanical investigation of softening of concrete loaded in compression. Heron 38, 5-94 (1993)

48. Warner, D.H., Molinari, J.F.: Micromechanical finite element modeling of compressive fracture in confined alumina ceramic. Acta Materialia 54(19), 5135-5145 (2006)

49. Weerheijm, J., Vegt, I., van Breugel, K.: Research developments and experimental data on dynamic concrete behaviour. In: Conference on Advances in Construction Materials, pp. 765-773 (2007)

50. Wittmann, F.H.: Structure of concrete with respect to crack formation. Fracture Mechanics of Concrete, Elsevier Science Publishers pp. 43-74 (1983)

51. Xu, X.P., Needleman, A.: Void nucleation by inclusions debonding in a crystal matrix. Modelling and Simulation in Materials Science and Engineering 1, 111-132 (1993) 